\title{
Influência dos aditivos sobre a permeabilidade e a velocidade de secagem de concretos refratários aplicados por projeção
}

\section{(Influence of additives on the permeability and drying behavior of wet shotcrete refractory castables)}

\author{
Y. A. Marques, D. Vasques Filho, R. G. Pileggi, V. C. Pandolfelli \\ Departamento de Engenharia de Materiais - DEMa \\ Universidade Federal de S. Carlos \\ Rod. Washington Luiz, km 235, C. P. 676, S. Carlos, SP, 13565-905 \\ g218375@polvo.ufscar.br,vicpando@power.ufscar.br
}

\begin{abstract}
Resumo
A projeção a úmido de concretos refratários consiste em aplicar o material como um jato de "spray" sobre a superfície, resultando numa elevada taxa de instalação e uma eficiente compactação do material. Esta técnica dispensa o uso de moldes devido à adição de aditivos aceleradores de pega e/ou coagulantes que reduzem rapidamente a fluidez do concreto projetado sobre a superfície. Usualmente, a definição do tipo e do teor de aditivo tem sido baseada unicamente na porcentagem de material aderido sobre a superfície e nas propriedades termomecânicas do concreto instalado, desconsiderando a secagem do material. Por esta razão, o presente trabalho teve como objetivo avaliar a influência de diferentes aditivos de projeção sobre a permeabilidade e a velocidade de secagem de concretos projetáveis de alta alumina. Os resultados obtidos identificaram uma acentuada influência do tipo de aditivo em relação ao risco de explosão durante a secagem, mesmo em baixas taxas de aquecimento.
\end{abstract}

Palavras-chave: concretos, refratários, projeção, aditivos, secagem.

\begin{abstract}
Wet shotcrete is a technique that consists on spraying the castable over a surface, allowing high placing rate and efficient material packing. Wet shotcrete does not require the use of molds due to the presence of accelerating admixtures or coagulants, which immediately reduce fluidity of the castable sprayed on the surface. The common choice of type and amount of additive has been based only on the percentage of material that adheres on the surface and on the thermomechanical properties of the castable after placing, without concerning about the drying behavior of the material. Thus, the purpose of this work was to evaluate the influence of shotcrete additives on the permeability and drying behavior of high alumina castables. The results show a great impact of the additives on the explosion risks during the drying process, even at low heating rates.
\end{abstract}

Keywords: castable, refractories, shotcrete, additives, drying.

\section{INTRODUÇÃO}

A indústria de refratários tem dedicado esforços na busca de concretos que aliem elevado desempenho termomecânico e alta taxa de secagem com reduzido custo de aplicação [1-6]. Nesse cenário, a projeção de concretos refratários a úmido ganha destaque por sua possibilidade de automação e melhor relação custo benefício em relação aos tradicionais concretos auto-escoantes e vibráveis.

O requisito básico da técnica de projeção consiste no bombeamento do concreto até o local de aplicação. Para que haja projeção, na saída da tubulação é acoplado um dispositivo (bocal de projeção) que possui uma entrada para ar comprimido de alta pressão, que acelera o concreto como um jato de "spray" sobre a superfície a ser recoberta. A consolidação do material sobre a superfície é proporcionada por um aditivo, responsável pela perda instantânea da fluidez do concreto, injetado juntamente no bocal de projeção $[1,5,6]$.

Existem diferentes aditivos que agem no concreto por diversos mecanismos. Os coagulantes atuam aumentando a força iônica ou mudando o pH do meio, influenciando assim a força de atração entre as partículas [1,7]. Outros são modificadores do meio líquido, formando géis que aprisionam a água do concreto promovendo a coesão do material [8]. Uma outra classe ainda forma ligações cruzadas entre as moléculas gerando uma rede tridimensional entre as partículas $[1,7]$.

Pela natureza dos diferentes mecanismos de atuação dos aditivos, a estrutura dos canais permeáveis pode ser afetada pela mudança no empacotamento das partículas ou ainda pela formação de produtos que preenchem estes canais. Como consequiência, a velocidade de secagem pode ser afetada e os riscos de explosão do concreto aumentados.

Apesar de estudos reológicos sobre a aplicação dos concretos de projeção e a influência dos diferentes mecanismos 
de coagulação terem sido documentados em literatura $[1,5$, 6], existem poucas pesquisas sobre sua influência na secagem do concreto após sua instalação. Deste modo, o presente trabalho teve como objetivo avaliar o impacto dos aditivos de consolidação sobre a permeabilidade e propriedades de secagem dos concretos de projeção.

\section{MATERIAIS E MÉTODOS}

Primeiramente foi formulada uma composição (Tabela I) de ultrabaixo teor de cimento baseada no modelo de Andreasen com coeficiente de empacotamento $q=0,26$ e com partículas na faixa de 0,1 a $4750 \mu \mathrm{m}$. O teor de água foi de $18 \%$ em volume, utilizando um policarboxilato éter (SKW - Alemanha) ( $0,15 \%$ em peso) como dispersante.

Tabela I - Composição do concreto de alta alumina $(q=0,26)$ utilizada no estudo da permeabilidade e secagem.

[Table I - High alumina castable composition $(q=0.26)$ for the evaluation of the permeability and drying behavior.]

\begin{tabular}{lcc}
\hline & Matérias-primas & \# volume \\
\hline \multirow{3}{*}{ Matriz $(<100 \mu \mathrm{m})$} & Aluminas Calcinadas & 17,0 \\
\cline { 2 - 3 } & ALO Branco 200/F & 16,0 \\
\cline { 2 - 3 } & Cimento CA14 & 2,0 \\
\hline Agregado $(<100 \mu \mathrm{m})$ & ALO Branco & 65,0 \\
\hline
\end{tabular}

Obs: Aluminas Calcinadas $=$ A1000 SG e A3000 FL; CA-14 = cimento de aluminato de cálcio; ALO Branco = alumina eletrofundida branca. Todas as matérias-primas foram fornecidas pela empresa Alcoa Alumínio S.A. A formulação do concreto foi elaborada utilizando o software PSDesigner [2].

[Note: Calcined alumina A1000 SG and A3000 FL; CA-14 = calcium aluminate cement; WFA = White fused alumina. The raw materials were supplied by Alcoa-Brazil and US. The formulation was calculated using the PSDesigner [2] software.]

A mistura do concreto foi realizada em um reômetro para concretos [9], assim como a injeção do aditivo, de maneira descrita a seguir:

A) homogeneização do concreto sob rotação constante de 33 rpm seguida da adição do teor exato de água para a virada.

B) adição do restante de água seguida da injeção do aditivo de projeção e homogeneização durante $20 \mathrm{~s}$.

C) moldagem sob vibração de corpos para análise de secagem (testes termogravimétricos) e permeabilidade, os quais foram curados por 24 horas em atmosfera saturada de vapor de água à $50^{\circ} \mathrm{C}$. Entre a etapa de cura e a execução dos ensaios, os corpos para análise termogravimétrica (ATG) foram selados e armazenados sob refrigeração $\left(8^{\circ} \mathrm{C}\right)$ para evitar a perda de umidade. Por sua vez, os corpos para permeabilidade foram secos a $110^{\circ} \mathrm{C}$, sendo posteriormente armazenados em sílica gel à temperatura ambiente.

\section{Aditivos}

Utilizando o procedimento experimental e a sequiência de ensaios descrita a seguir, três aditivos (sulfato de alumínio (Aldrich - Brasil), diacetato de alumínio (Aldrich-Alemanha) e poliacrilato de sódio (Basf - Alemanha)) foram avaliados em dois teores distintos ( 0,2 e $0,4 \%$ em peso) (Tabela II). Além disso, sistemas com $0,025 \%$ em peso de alginato de sódio em adição ao poliacrilato de sódio (Fluka - Suíça) $(0,2 \%$ e 0,4\%), foram também estudados.

Tabela II -Aditivos avaliados quanto a sua influência nas propriedades de secagem e permeabilidade do concreto $[1,5,7,9]$.

[Table II - Additives used to evaluate their effect on the permeability and drying behavior of the castables [1, 5, 7, 9].]

\begin{tabular}{lc}
\hline ADITIVOS & $\begin{array}{c}\text { MECANISMO DE } \\
\text { COAGULAÇÃO }\end{array}$ \\
\hline Sulfato de Alumínio & $\uparrow$ força iônica \\
\hline Diacetato de Alumínio & $\uparrow$ força iônica $+\downarrow \mathrm{pH}$ \\
\hline Poliacrilato de Sódio & † força iônica + "bridging" \\
& "depletion" \\
\hline Alginato de Sódio & formação de gel + ligações cruzadas \\
\hline
\end{tabular}

Foram selecionados aditivos com princípios distintos de coagulação para a consolidação do concreto (Fig. 1). O sulfato de alumínio (SA) e o diacetato de alumínio (DIAC) são aditivos inorgânicos que não contém álcalis, e o sulfato de alumínio com amplo uso industrial. Basicamente, estes aditivos aumentam a força iônica do sistema, alterando o balanço de energia potencial e promovendo atração e aglomeração das partículas [1].

O poliacrilato de sódio (PAS) utilizado neste trabalho é um polieletrólito orgânico com elevado peso molecular $(15000 \mathrm{~g} / \mathrm{mol})$
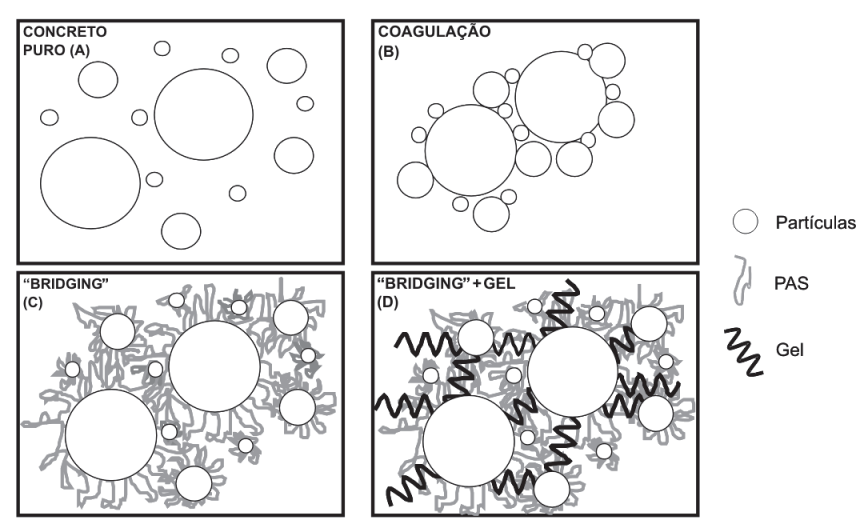

Figura 1: Mecanismos de Coagulação do Concreto: (A) Concreto sem aditivos. (B) Aglomeração devido à atração entre as partículas. (C) Efeito de "bridging" causado pelas moléculas de poliacrilato de sódio (PAS). (D) "Bridging" e formação de gel pelo alginato de sódio.

[Figure 1: Coagulation Mechanisms in the concrete: (A) Concrete without additives. (B) Aglomeration due to attraction forces among the particles. (C) Bridging effect caused by the sodium polyacrylate (PAS) molecules. (D) Bridging effect and gel formation promoted by the alginic acid sodium salt.] 
que coagula as partículas em suspensão por mecanismos de "bridging", "depletion" e aumento na força iônica [10]. Contudo, o efeito estérico associado às suas moléculas impede que as partículas se aproximem em demasia, preservando a posição original das mesmas.

O alginato de sódio (Alg) é um polímero de alto peso molecular derivado das algas marinhas marrons $[7,8]$. Seu princípio de atuação é baseado na formação de um gel com a água, formando ligações cruzadas com os íons de cálcio dissolvidos a partir do cimento. Desta forma, a consolidação promovida pelo alginato não provoca a modificação da posição das partículas em suspensão.

\section{Permeabilidade}

Os corpos de prova secos a $110{ }^{\circ} \mathrm{C}$ utilizados para avaliar a permeabilidade do concreto (cilindros $\mathrm{D}=60 \mathrm{~mm}$ e $\mathrm{h}=22 \mathrm{~mm}$ ) foram submetidos a testes conduzidos em temperatura ambiente seguindo o procedimento descrito em [3].

Constantes de permeabilidade foram obtidas pela equação de Forchheimer, expressa para escoamento de fluidos compressíveis $[2,3]$ :

$$
\left(\frac{\mathrm{P}_{\mathrm{i}}^{2}-\mathrm{P}_{0}^{2}}{2 \mathrm{P}_{0} \mathrm{~L}}\right)=\frac{\mu}{\mathrm{k}_{1}} \mathrm{v}_{\mathrm{s}}+\frac{\rho}{\mathrm{k}_{2}} \mathrm{v}_{\mathrm{s}}^{2}
$$

onde $\mathrm{P}_{\mathrm{i}}$ e $\mathrm{P}_{0}$ são, respectivamente, a pressão absoluta na entrada e saída do corpo de prova, $\mathrm{v}_{\mathrm{s}}$ é a velocidade do fluido, $\mathrm{L}$ é a espessura do corpo, $\mu$ a viscosidade do ar e $\rho$ a densidade do ar. Os parâmetros $\mathrm{k}_{1}$ e $\mathrm{k}_{2}$ são respectivamente as constantes de permeabilidade Darciana e não-Darciana [2, 3].

\section{Secagem}

Para avaliar a velocidade de secagem das composições, testes foram realizados em uma balança termogravimétrica desenvolvida especialmente para concretos [3]. Neste ensaio, o corpo de prova (cilindros de diâmetro $(\mathrm{D})=$ altura $(\mathrm{h})=$ $40 \mathrm{~mm}$ ) é suspenso no interior de um forno permitindo que o calor alcance, simultaneamente, todas as faces do corpo. Dados da temperatura do forno e da perda de massa da amostra são registrados por um computador em intervalos de $5 \mathrm{~s}$. A taxa de aquecimento foi fixada em $10{ }^{\circ} \mathrm{C} / \mathrm{min}$ para uma faixa de temperatura de 30 a $600{ }^{\circ} \mathrm{C}$.

A perda de massa durante a secagem foi avaliada pelo parâmetro $\mathrm{W}$ que quantifica a fração acumulada de água expelida durante $\mathrm{o}$ aquecimento pela quantidade inicial contida no concreto, variando de 0 a $100 \%$. W é definido pela equação a seguir:

$$
W(\%)=x 100\left(\frac{M_{o}-M}{M_{o}-M_{f}}\right)
$$

onde $\mathrm{M}$ é a massa instantânea registrada para um tempo $\mathrm{t}$ durante o aquecimento, $\mathrm{M}_{0}$ é a massa inicial e $\mathrm{M}_{\mathrm{f}}$ a massa final do corpo-de-prova.

A taxa experimental de secagem do concreto foi obtida através da derivada que expressa a perda de massa em função do tempo (dW/dt), definida por:

$$
\left(\frac{d W}{d t}\right)=\frac{W_{i+1}-W_{i-1}}{t_{i+1}-t_{i-1}}
$$

\section{RESULTADOS E DISCUSSÃO}

\section{Permeabilidade do corpo seco}

Os resultados demonstraram que os diferentes aditivos de projeção afetaram distintamente a permeabilidade do concreto (Fig. 2), sendo possível diferenciar a atuação dos aditivos inorgânicos e dos orgânicos.

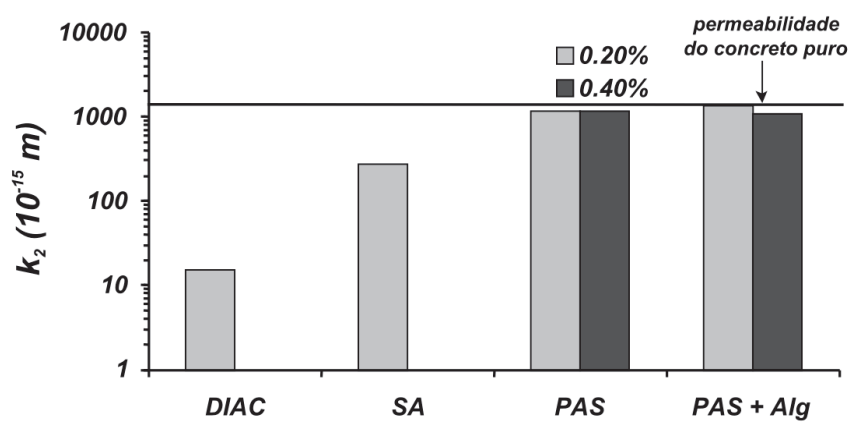

Figura 2: Influência dos aditivos de projeção sobre a constante não-Darciana $\left(\mathrm{k}_{2}\right)$ de permeabilidade do concreto.

[Figure 2: Influence of the shotcrete additives on the non-Darcian permeability constant $\left(k_{2}\right)$ of the castable.]

No teor de $0,2 \%$, os aditivos inorgânicos (sulfato de alumínio e diacetato de alumínio) reduziram a permeabilidade $\left(\mathrm{k}_{2}\right)$ das composições a níveis inferiores ao do concreto puro, sendo esta diminuição mais intensa com o uso do diacetato. Este resultado pode ser atribuído à compressão dos poros permeáveis devido ao mecanismo de aglomeração (aumento na força iônica e/ou alteração no $\mathrm{pH}$ ) induzido por estes compostos químicos.

$\mathrm{O}$ aumento no teor destes aditivos para $0,4 \%$ intensificou a ação coagulante dos mesmos, de forma que não foi possível moldar corpos de prova homogêneos e isentos de macro defeitos (trincas e buracos) para a análise de permeabilidade. Como conseqüência, os elevados valores de permeabilidade obtidos com $0,4 \%$ dos aditivos inorgânicos não puderam ser considerados, pois estes defeitos geram canais preferenciais para o fluxo do ar durante o ensaio.

Por sua vez, os aditivos orgânicos, poliacrilato de sódio $(0,2$ e $0,4 \%)$ e as combinações de poliacrilato de sódio $(0,2$ e $0,4 \%)$ e alginato de sódio $(0,025 \%)$, não influenciaram 
significantemente a permeabilidade do concreto, apresentando valores similares ao do material puro. Estes resultados estão de acordo com o mecanismo de consolidação descrito para estes aditivos, o qual não promove a aproximação entre as partículas, mantendo no sistema a estrutura original do concreto puro.

\section{Secagem}

Os perfis de secagem dos concretos úmidos (curados a $50^{\circ} \mathrm{C}$ ) podem ser analisados através das curvas da porcentagem de perda de massa (W) durante o aquecimento em função da temperatura do forno (Fig. 3).

Comparando-se o concreto sem aditivos (puro) com os aditivados, pode-se observar que o puro apresentou um perfil de secagem mais rápido que os demais, tendo perdido $50 \%$ de sua água em aproximadamente 24 minutos, com a temperatura do forno em torno de $270{ }^{\circ} \mathrm{C}$.

Para o teor de $0,2 \%$, todos os aditivos apresentaram um maior tempo ( $>25$ minutos) para perda deste mesmo percentual de água (50\%), e como consequiência alcançaram uma maior temperatura $\left(>280{ }^{\circ} \mathrm{C}\right)$. Comportamento semelhante foi verificado também no teor de $0,4 \%$ de aditivo, sendo então
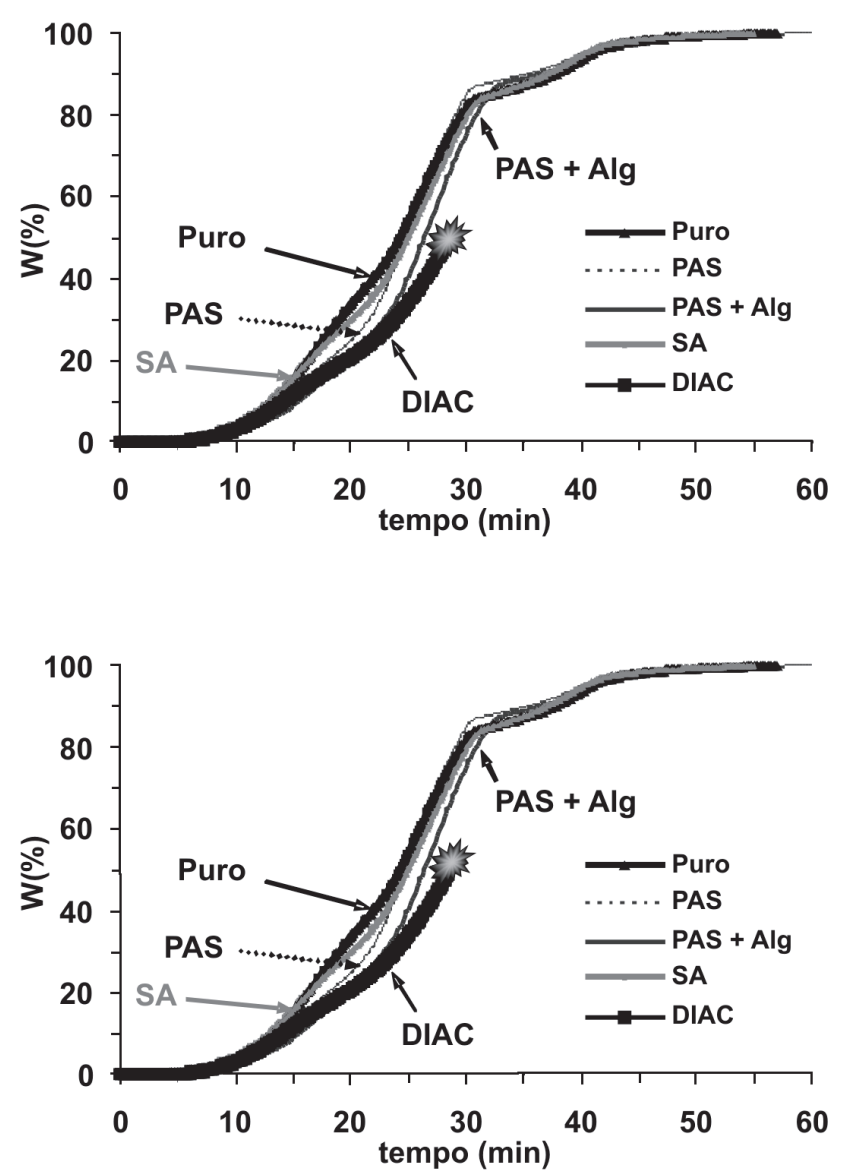

Figura 3: Perfis de secagem para o concreto puro e com $0,2 \%$ em peso dos diferentes aditivos de projeção testados: (A) W x tempo; (B) W x temperatura do forno. Obs: indica explosão do corpo.

[Figure 3: Drying curves for the castable without additives and with 0.2 wt. \% of the shotcrete additives: (A) Wx time; (B) Wx furnace temperature. Note: indicates sample's explosion.] possível afirmar que a introdução dos aditivos retardou, de algum modo, a saída de água no concreto.

$\mathrm{O}$ impacto sobre a secagem foi marcante para o diacetato de alumínio ( $0,2 \%$ DIAC), o qual retardou de tal maneira o processo de saída da água, a ponto de provocar a explosão do corpo. Esse resultado pode estar associado com a redução na permeabilidade do concreto induzida por este aditivo (Fig. 2), a qual dificultou a saída de água, provocando sua pressurização no interior do corpo acima do limite de resistência mecânica do mesmo.

A Fig. 4 apresenta a taxa de secagem (dW/dt) dos concretos em função da temperatura do forno. O primeiro pico em cada curva representa a saída de água livre por evaporação. Já o segundo pico está relacionado à perda de massa por ebulição da água. O terceiro pico indica a saída da água dos hidratos formados pela reação do cimento com a água $[2,3]$.

Quanto maior a taxa de secagem na região de evaporação, menor o risco de explosão, pois a pressão de vapor nesta faixa de temperatura é ainda baixa, não causando danos à estrutura do corpo. Contudo, a saída da água por ebulição resulta na sua pressurização nos poros da estrutura, podendo eventualmente atingir a tensão critica de ruptura do material.
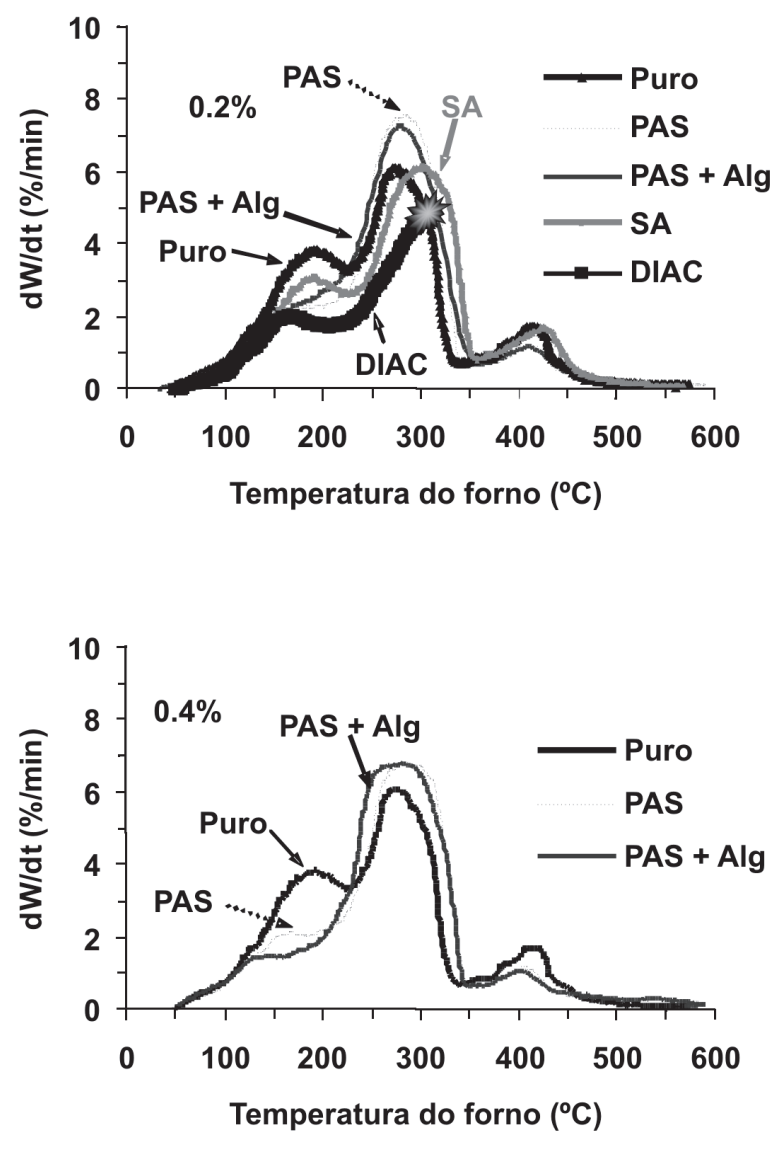

Figura 4: Curvas de secagem (dW/dt $\mathrm{x}$ temperatura do forno) para o concreto com diferentes aditivos: (A) teor de $0,2 \%$ em peso e (B) teor de $0,4 \%$ em peso, comparados ao concreto puro. Obs: indica a explosão do corpo. [Figure 4: Drying profiles (dW/dt $x$ furnace temperature) for the castable with different additives: (A) 0.2 wt. \% and (B) $0.4 \mathrm{wt} . \%$. Note: indicates sample's explosion.] 
Sendo assim, o maior pico na região de evaporação do concreto puro pode indicar que este apresenta menor risco de explosão que os das composições sob ação dos aditivos $(0,2$ e $0,4 \%$ ), uma vez que todos dificultaram a saída de água nesta faixa de temperatura. Além disso, esta maior facilidade para saída de água na região de evaporação reduziu a taxa de secagem do material puro na ebulição, devido à menor quantidade residual de líquido a ser eliminada nesta etapa.

Em relação aos aditivos inorgânicos $(0,2 \%)$, a intensa compressão dos poros da estrutura pela coagulação das partículas durante a moldagem resultou em uma baixa permeabilidade e assim em um menor pico de evaporação. No caso do diacetato de alumínio (DIAC), essa reduzida permeabilidade foi provavelmente responsável pela explosão verificada na temperatura aproximada de $338^{\circ} \mathrm{C}$.

O sulfato de alumínio (SA) também prejudicou a secagem do concreto (Fig. 3) pela redução da permeabilidade, o que além de diminuir a taxa de saída de água na evaporação, deslocou o pico de perda de água por ebulição para maiores temperaturas e aumentou a probabilidade de ocorrência da explosão. Infelizmente, o impacto do aumento no teor destes aditivos $(0,4 \%)$ sobre a taxa de secagem não pôde ser avaliado devido à rápida pega que eles proporcionaram, impossibilitando a produção de corpos de prova.

Por sua vez, os aditivos orgânicos $(0,2$ e $0,4 \%$ de poliacrilato de sódio com ou sem adição de $0,025 \%$ de alginato de sódio), também afetaram negativamente a saída de água do concreto na região de evaporação e na ebulição a taxa de secagem foi elevada acima do nível do material puro e deslocada para temperaturas maiores. Convém ressaltar que ambas alterações no perfil de secagem também elevam o risco de explosão em relação ao material puro.

Uma vez que estes aditivos orgânicos pouco afetam a estrutura de empacotamento das partículas, este comportamento indica que as moléculas do poliacrilato e do alginato de algum modo dificultaram a saída da água do sistema na região de evaporação.

Estudos mais aprofundados são ainda necessários para melhor compreender como otimizar o emprego dos aditivos orgânicos em concretos projetáveis.

Pode-se então afirmar que os aditivos inorgânicos apresentaram maior risco de explosão, uma vez que dificultaram a saída da água em ambas regiões da secagem, enquanto os orgânicos predominantemente afetaram a evaporação.

Do ponto de vista das aplicações reais, onde o volume de material é maior e as geometrias das estruturas de concreto são mais complexas, as alterações nos perfis de secagem dos materiais são ainda mais sensíveis, elevando os riscos de explosão [3]. Portanto, um outro critério para a seleção de aditivos de projeção, além do nível de rebote [1], deve ser o efeito sobre a secagem.

\section{CONCLUSÕES}

A introdução dos aditivos de projeção influenciou a permeabilidade e a secagem do concreto. $\mathrm{O}$ concreto puro apresentou um perfil de secagem mais rápido, com maior saída de água por evaporação.

Aditivos inorgânicos que induzem a aglomeração das partículas (sulfato de alumínio e diacetato de alumínio) reduziram a permeabilidade do concreto para teores de $0,2 \%$ em peso. O sulfato e, mais intensamente, o diacetato, retardaram a secagem do concreto aumentando os riscos de explosão, sendo que o último causou a explosão do material para o teor de $0,2 \%$.

Os aditivos orgânicos (poliacrilato de sódio e alginato de sódio) não alteraram significativamente a permeabilidade do material seco. Apesar de retardarem a secagem do concreto, estes aditivos apresentaram menores riscos de explosão, quando comparados com os inorgânicos. São, portanto, mais indicados para utilização em concretos refratários aplicados por projeção a úmido, principalmente se nas composições contendo estes aditivos fibras orgânicas forem incorporadas.

\section{AGRADECIMENTOS}

Os autores agradecem à FAPESP, CNPq e à ALCOA Alumínio S.A. pelo apoio dado à execução deste trabalho. Além disso, os autores agradecem aos pesquisadores Murilo Innocentini, Fábio Cardoso e Rafael Salomão pelas suas colaborações.

\section{REFERÊNCIAS}

[1] R. G. Pileggi, Y. A. Marques, D. Vasques Filho, A. R. Studart, V. C. Pandolfelli, Wet-Shotcrete Additives, Am. Ceram. Soc. Bull. 81, 61 (2002) 51-57.

[2] M. D. M. Innocentinni, R. G. Pileggi, F. T. Ramal Jr., V. C. Pandolfelli, "Permeability and drying behavior of PSD-designed refractory castables", Am. Cer. Soc. Bull. (2003) aceito.

[3] M. D. M. Innocentinni, A. R. F. Pardo, V. C. Pandolfelli, "Permeability of high alumina refractory castables based on various hydraulic binders", J. Am. Ceram. Soc. 85, (2001) 1517-1521.

[4] M. D. M. Innocentinni, A. R. Studart, R. G. Pileggi, V. C. Pandolfelli, "How PSD affects the permeability of castables", Am. Ceram. Soc. Bull. 80, 5 (2001) 31-36.

[5] M. Jolin, D. Beaupré, S. Mindess, "Tests to characterize properties of fresh dry-mix shotcrete", Cement Concrete Res. 29, 5 (1999) 753-760.

[6] C. Paglia, F. Wombacher, H. Bohni, "The influence of alkali-free and alkaline shotcrete accelerators within cement systems", Cement Concrete Res. 31, 6 (2001) 913-918.

[7]A. R. Studart, V.C. Pandolfelli, E. Tervoot, L. J. Gauckler, "Gelling of alumina suspensions using alginic acid salt and hydroxyaluminum diacetate", J. Am. Ceram. Soc. 85, 11 (2002) 2711-2718.

[8] K. H. Khayat, "Viscosity enhancing admixtures for cementbased materials: An overview," Cement Concrete Comp. 20, 2-3 (1998) 171-188.

[9] R. G. Pileggi, A. E. Paiva, J. Gallo, V. C. Pandolfelli, "Novel rheometer for refractory castables", Am. Ceram. Soc. Bull. 78, 5 (2000) 54-58.

[10] I. R. Oliveira, A. R. Studart, R.G. Pileggi, V.C. Pandolfelli, Dispersão e empacotamento de partículas: princípios básicos e aplicações em processamento cerâmico, Fazendo Arte Editorial, São Paulo (2000) 224 p.

(Rec. 04/06/03, Ac. 15/07/03) 